\title{
Intelligent Agent Supported Flexible Workflow Monitoring System
}

\author{
Minhong Wang and Huaiqing Wang \\ Department of Information Systems, City University of Hong Kong \\ Kowloon, Hong Kong \\ \{iswmh, iswang\}@is.cityu.edu.hk
}

\begin{abstract}
The unpredictability of business process requires workflow systems to support workflow monitoring functions with the ability to flexibly adapt to the changing environment. Traditional approaches to handling this problem have fallen short, providing little support for monitoring flexibility. In this paper, we will describe a flexible workflow monitoring system, in which a society of intelligent agents work together to perform flexible monitoring processes based on various monitoring requests.
\end{abstract}

\section{Intelligent Agent Assistance}

With the rising tide of unexpected output of tasks, events or exceptions due to changing environment to be met with during the whole life of an organization, the reinforcement of workflow adaptability has become crucial to cater for these deviations [1]. It is important for exception management to have the ability to detect an actual exception or the possibility of an exception as well as improve the ability of participant to react and recover. However, present workflow systems provide only a few built-in monitoring functions, not allowed changes of monitoring specifications [2]. Our proposed monitoring system is to develop a flexible monitoring application, in which various monitors can execute respective monitoring tasks by submitting their monitoring requests on workflow management. It is our belief that intelligent agents are well suited to dealing with problems of flexible monitoring in an autonomous and collaborative fashion. By utilizing a society of intelligent agents, each charged with carrying out a different function autonomously, such as monitoring request processing, monitoring scheming, data searching and diagnosing, our workflow monitoring system can be applied to perform monitoring tasks in a flexible way.

Intelligent agents, which used to denote software-based computer systems that enjoy such properties as autonomy, co-operativity, reactivity, pro-activity and mobility [3], are well suited to dealing with flexible monitoring in our system. The proposed system consists of a number of agents, which operate autonomously and cooperate with each other to perform their tasks. The taxonomy of intelligent agents 
with specific behaviours is shown in Fig.1. In our system, intelligent agents can be classified into the following five sub-classes in terms of their functions.

User Agent. User Agent assists monitors to input various monitoring requests, receive monitoring results and communicate with other monitors for further decision.

Monitoring Scheming Agent. This agent is charged to process monitors' requests, generate respective monitoring schemes, and send them to Searching Agents and Diagnostic Agent to perform data searching and diagnosing respectively.

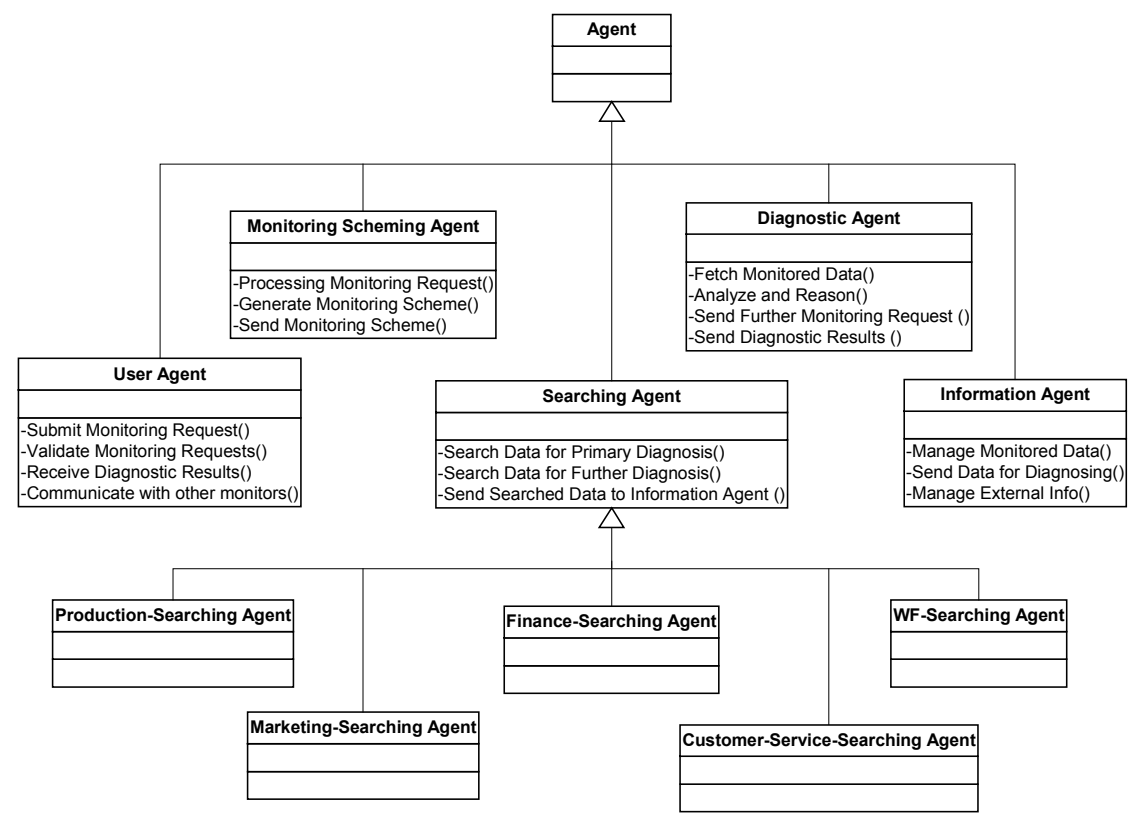

Fig.1. A class diagram of intelligent agents in the workflow monitoring system

Searching Agent. There are various searching agents in our system, such as Workflow Searching Agent, Production Searching Agent, Marketing Searching Agent, and so on, which may be located distributedly. Each assists to collect monitored data in workflow systems or other application systems for various monitoring schemes.

Diagnostic Agent. The role of this agent is to make analysis for various monitoring schemes, and then distribute diagnostic results such as warning messages or workflow adjustment indications to monitors or a workflow system. Diagnoses are made on the basis of diagnostic rules specialized in monitoring schemes, as well as diagnostic policies stored in Diagnostic Agent as meta level knowledge for diagnosis.

Information Agent. This agent is responsible for information relative management for monitoring processes, such as information store, retrieval or filtration on monitored data, monitoring reports, other monitors' information, industry information or other useful information supporting monitoring. 


\section{System Architecture}

Based on the above taxonomy of intelligent agents, we present the architecture of our intelligent agents based workflow monitoring system in Fig.2. This framework provides the conceptual basis for thinking about the overall monitoring system.

As an independent monitoring system, our system contains two kinds of interactions with outside. First, various monitors interact with the monitoring system via User

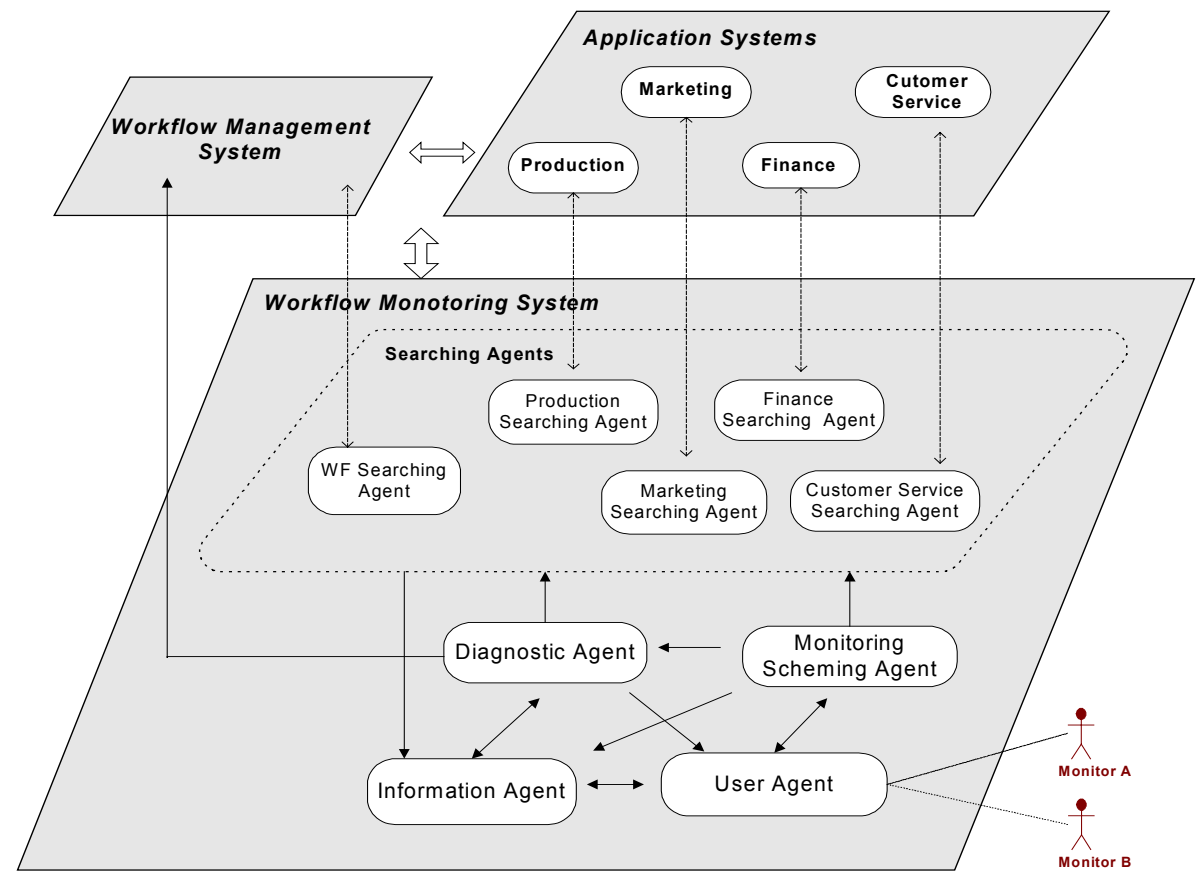

Fig.2. An architecture of intelligent agents based workflow monitoring system

Agents to search available monitored data lists, submit monitoring requests, receive warning messages, browse monitoring reports, as well as communicate with other monitors for further decision. Second, our monitoring system interacts with a workflow system or other application systems of an organization to collect necessary information for monitoring as well as perform adjustments on the workflow system. The operation inside the monitoring system is described in the following section.

\section{System Operation}

In order to evaluate the effectiveness of our proposed workflow monitoring system, we will describe its operation (see Fig.3) based on an example of a monitoring process to demonstrate how these intelligent agents work together. 
For example, a monitor is to monitor components supply for current production. He submits his monitoring request including monitored data list (e.g. order status, available time, quantity in storage) and diagnostic rules (e.g. order status rule, minimum storage rule) via User Agent to Monitoring Scheming Agent. Then, Monitoring Agent transforms this monitoring request into a monitoring scheme after interpretation and validity checking on it, and sends the monitoring scheme to relative Searching Agents and Diagnostic Agent. After receiving the monitoring scheme, Search Agents will collect relative data for preliminary diagnosis, and send them to Information Agent as well as send a data-available notice to Diagnostic Agent. Once receiving the notice, Diagnostic Agent will fetch those data from Information Agent, and make preliminary diagnosis based on diagnostic rules specialized in the monitoring scheme. If the result of preliminary diagnosis is abnormal (e.g., a component order has kept being processed for 2 hours with no reply), Diagnostic Agent will send a further monitoring request (e.g. to monitor detail processing information of this order) to Search Agents. This transform from preliminary monitoring to further monitoring is controlled by a diagnostic policy stored in Diagnostic Agent. Subsequently, further data are collected and a further diagnosis is made. At last, Diagnostic Agent generates a monitoring report including workflow adjustment indications (e.g. activate a new order for a type of component) or warning message (e.g. a type of component is going to be out of supply). Workflow adjustment indications are sent to the workflow system, and warning messages are sent to monitors through User Agents. Also, monitors can browse detail monitoring reports or monitoring histories via User Agents, as well as communicate with other monitors for further decision.

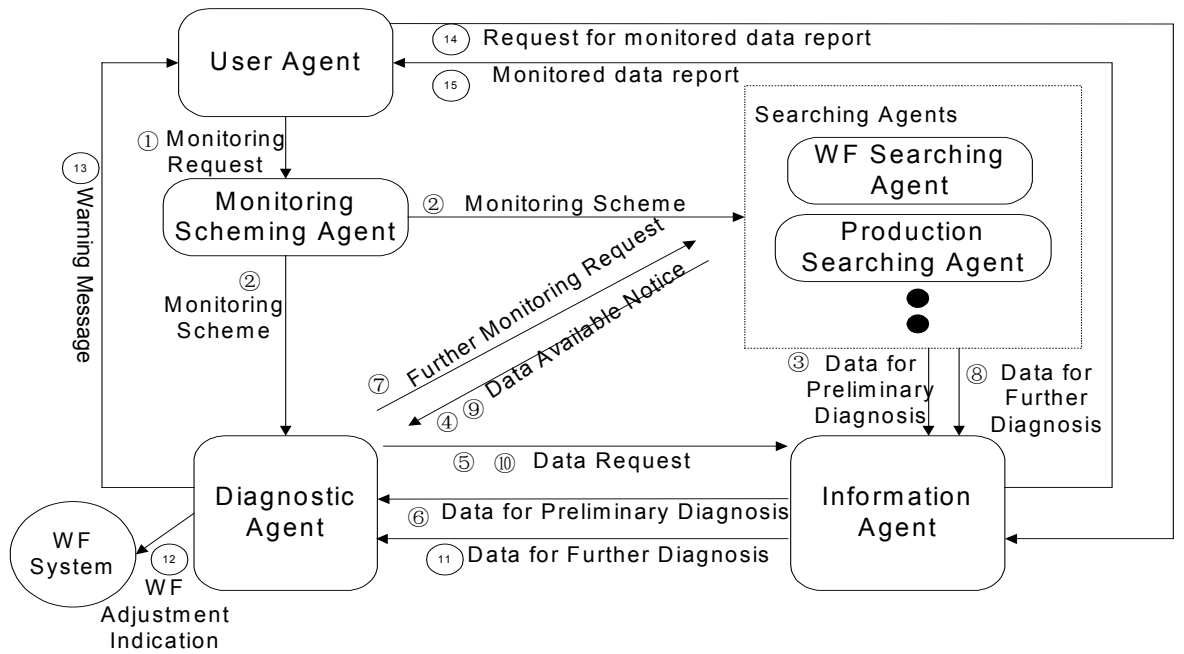

Fig.3. Intelligent agents based flexible monitoring operation 


\section{References}

1. Kammer, P.J., Bolcer, G.A., Taylor, R.N., Hitomi, A.S., Bergman, M., Techniques for Supporting Dynamic and Adaptive Workflow, Computer Supported Cooperative Work (CSCW), November 2000, pp.269-292.

2. Baker, D.; Georgakopoulos, D.; Schuster, H.; Cassandra, A.; Cichocki, A., Providing customized process and situation awareness in the collaboration management infrastructure, Cooperative Information Systems, 1999. CoopIS '99. Proceedings. 1999 IFCIS International Conference on, 1999, pp.79 -91.

3. Wang, H., "Intelligent Agent Assisted Decision Support Systems: Integration of Knowledge Discovery, Knowledge Analysis, and Group Decision Support", Expert Systems with Applications, 1997, Vol. 12, No. 3, pp.323-335. 